\title{
The Joint External Evaluation process in Cameroon: Assessing the country's capacity for health security
}

Viviane FOSSOUO NDOUNGUE ( $\nabla$ vfossouo@gmail.com )

Ministere de la Sante Publique https://orcid.org/0000-0001-9521-3380

Mohamed Moctar Mouiche Molium

Infectious diseases detection and surveillance project Cameroon

Christie Tiwoda

Ministere de la Sante Publique

Oumarou Gnigninanjouena

Ministere de la Sante Publique

Serge Alain Sadeuh-Mba

Centre Pasteur du Cameroun

Roland Kimbi Wango

Organisation mondiale de la Sante

Arouna Njayou Ngapana

Universite des Montagnes Faculte des Sciences de la Sante

Research note

Keywords: International Health Regulation, Joint External Evaluation, Health security capacity, Cameroon

Posted Date: October 7th, 2019

DOI: https://doi.org/10.21203/rs.2.15807/v1

License: (c) (i) This work is licensed under a Creative Commons Attribution 4.0 International License.

Read Full License 


\section{Abstract}

Objectives The objective of this study was to present the JEE process in Cameroon as well as Cameroon's capacities to manage public health threats in accordance with the IHR 2005. Cameroon JEE process and data for the 48 indicators within the 19 technical areas was examined.Results Cameroon's overall score was $1.98 \pm 0.93$ and $48 / 34$ indicators $(70.38 \%)$ had scores less than 2 on a 1 to 5 scale. In the "Detect" category the average score was 2.7. Technical areas with the lowest average scores were Reporting and National laboratory system. The weakest indicators in the "Prevent" category were antimicrobial resistance, biosafety and biosecurity, and National legislation, policy and financing. Emergency Response Operations, Preparedness, Medical Countermeasures and Personnel Deployment have the lowest scores in the "Respond" category. Chemical Events and Points of Entry have the lowest score in "Other IHRrelated hazards and Point of Entry". Scores attributed during the country self-assessment were $73 \%$ similar to those of the JEE process.

\section{Introduction}

Health security in Cameroon has been marked over the past 50 years by several events related to infectious diseases such as: i) imported cases of measles and wild polio - viruses leading to epidemics requiring several vaccine responses campaigns, ii) Cholera epidemics associated to imported cases which started in the northern region and later affected many other regions of Cameroon in 2012 and 2018. Cameroon also faced the emergence of zonooses such as avian influenza outbreaks in 2006 and 2016 and monkey pox in 2017. Other major emergencies in Cameroon included chemical intoxication of humans and animals by gas emissions from Lake Nyos in 1986 in Bamenda, the volcanic eruptions in the south West region in 1999 and 2012. Food safety related issues such as importation of contaminated infant milk and listeria-contaminated ham were also reported.

Given universal vulnerability to these threats, the World Health Organization (WHO) established the International Health Regulations (IHR) (2005), which requires all countries to develop and strengthen their capabilities to detect, assess, report, and respond to public health events through the development of core capacities,[1], thus, limiting the international spread of diseases and contributing to the reduction of the international impact of public health emergencies. After the entry into force of the IHR (2005) in 2007, States Parties had 5 years, until 2012, to develop the core capacities of the IHR. An additional period of 2 years was granted upon presentation of an action plan,[2]. In 2014, only about $30 \%$ of States Parties had met the required capacities. In 2015, the WHO recommended that "Countries move from exclusive selfassessment to approaches that combine self-evaluation, peer review, and voluntary external evaluations involving a combination of domestic and independent experts"[2]. In that perspective, WHO developed the Joint External Evaluation (JEE) process and JEE tool in February 2016 as part of the IHR (2005) Monitoring and Evaluation framework as one of the tools to determine countries' capacity to prevent, detect and response to public health threats in addition to After Action Review and simulation exercises, [3]. The objective of this study was to present the JEE process in Cameroon as well as Cameroon's 
capacities to prevent, detect and respond to public health threats in accordance with the International Health Regulation 2005.

\section{Method}

A descriptive cross sectional approach was used to outline the JEE process in Cameroon. Excel was used to extract data and the R.3.4.3 software was used to analyze and generate results. The overall average of Cameroon's score was calculated. The indicators were grouped according the scores obtained during the JEE process. The median score was obtained and presented with respect to the main categories used in the JEE tool: Prevent, Detect, Respond, Other IHR related hazards and Points of Entry. Then, the mean was obtained for the 19 technical areas. Afterwards, the difference between the self-assessment scores and the JEE scores was presented. Data interpretation was done for each JEE tool indicator in relation to different characteristics reflecting their capacity level, based on an incremental likert scale criteria ranging from 1 to 5 ( 1 = no capacity, 2 = limited capacity, 3 = developed capacity, $4=$ demonstrated capacity, and 5 = sustainable capacity.)

\section{Results}

Out of the 48 indicators, $34(70.38 \%)$ had scores less than 2 on a scale of 1-5 (Fig 1).

Figure 1: Figure 1: Number of indicators per score during the JEE $(n=48)$

In "Prevent" category, scores varied from 1 to 3 with half of the 15 indicators having a score below 2. In "Detect" category, the scores ranged from 1 to 4 with half of the13 indicators having a score below 3 . Concerning the "Respond" category, the scores ranged from 1 to 3 with half of the 14 indicators having a score below 2. Finally, in "Other IHR-related hazards and Point of Entry" category, scores ranged from 1 to 3 with half of the 6 indicators having a score lower than 2 (fig 2).

Figure 2: Dispersion of the JEE Indicators score of each domain

In "Prevent" category, average score was 1.8 and technical areas with the lowest average scores being Antimicrobial resistance (AMR), training and practices in the field of biosafety and biosecurity, and National legislation, policy and financing with a score of 1 each. In "Detect" category the average score was 2.7 and technical areas with the lowest average scores being Reporting and National laboratory system with a score of 2 and 2.2 respectively. Concerning "Respond" category, average score was 1.8 and technical areas with the lowest average score being Preparedness, Medical Countermeasures and Personnel Deployment, and Emergency Response Operations with average scores of 1.5, 1.5, and 1.8 respectively. Finally, concerning "Other IHR-related hazards and Point of Entry" category, average score was 1.3 and technical areas with the lowest average score were Chemical Events and Points of Entry with score of 1 each (fig 3).

Figure 3: Summary of the JEE average score per technical areas $(n=19)$ 
Further analysis showed that between the self-assessment and JEE scores, there was a variation in score on only 13 of the 48 indicators measured, i.e. $72.91 \%$ of the scores concordant between national and external experts. Only two indicators had an increase in their scores: the indicator measuring capacity related to the availability of veterinary and animal health workforce, and the indicator measuring capacity related to public communication.

\section{Discussion}

Cameroon's overall score was $1.98 \pm 0.93$. Regarding the difference in score between the self-assessment and the JEE, there was a variation on only $27.9 \%$ of the indicators. Establishing a strong multidisciplinary team contributed to this performance. This reflects the transparency and objectivity with which the Cameroonian government conducted this self-assessment. Decreasing scores were those for which national actors did not have a good interpretation of questions for some indicators.

While must scores varied by only one point, the score of indicator "relationship between the services in charge of security and those in charge of public health when managing a public health event", reduced by 2 points. This indicator is measured by the existence of a memorandum of understanding or other agreement between the public health sector and the security authorities. But in Cameroon, the role of the Civil Protection Department is to ensure multisectoral coordination of the response during events involving several sectors. As a result, the signing of collaboration agreements between public administrations is not often observed.

In general, Cameroon's scores are nearly $70 \%$ below 2 . In the field of "prevention", scores vary from 1 to 3 . Non-existing capacities are related to the absence of a systematic multi-sectoral analysis of legislation related to IHR (2005) implementation using the "One Health" approach in order to make recommendations to the government and allow necessary updates. Training and practices in the field of biosafety and biosecurity were also identified as non-existing capacities since aspects were somewhat restricted to refering human and animal laboratories at Central level and there was neither survey on the training needs nor a national plan for staff trainings in these domains. Moreover, the absence of an AMR surveillance system does not make it possible to control this major public health threat. Strengthening laboratory technical platforms for detection of AMR and the development of a national plans for detection and notification of AMR and prevention and infection control programs could help to pave the way to fight AMR in Cameroon.

Regarding detection, scores vary from 1 to 4 . The non-existent capacities concerned the absence of a harmonized and secured sample transport/transfer system and standard operating procedures at all levels. The establishment of an intersectoral network of human, animal and environmental health laboratories, including food safety, could help to bridge this gap. Lack of a network and notification protocols was also identified as non-existing capacity. It is therefore essential to develop and disseminate to all members of the National IHR Focal Point standard operating procedures for the approval and notification to WHO, OIE or FAO of events likely to constitute Public Health Emergency of International 
Concern (PHEIC) in a "One Health" approach. However, Cameroon has demonstrated capacities: (i) laboratory analyses for the detection of priority diseases; (ii) syndromic surveillance; (iii) training in basic and advanced field epidemiology.

Concerning response, scores ranged from 1 to 3 . Non-existing capacities were the absence of a national multi-hazard public health emergency preparedness and response plan, weak capacity to activate emergency responses, absence of operational plans and procedures at the PHEOC, absence of a system for sending and receiving health personnel during a public health emergency and absence of risk communication systems. With the support of the GHSA, a PHEOC Center has been temporarily set up within the department in charge of epidemiological surveillance while awaiting the dedicated building. The PHEOC was activated prior to the JEE to respond to health emergencies such as the train derailment in Eseka, the avian influenza epidemic and the 2016 Women's African cup of nations. However, absence of regulatory texts for the creation and operation of the PHEOC and its activation only when a public health event occurs has strongly contributed to maintaining the capacity as non-existing despite these activations.

For other IHR-related risks and points of entry component, the scores recorded were the lowest, ranging from 1 to 2 . While they are directly concerned by potential dangers, non-existent capacities are those related to the absence of the main capacities at the 35 designated entry points (airports, international ports and ground crossings). Although Cameroon ratified important international conventions on the disposal of hazardous waste, there is no coordination system, committee or body in place to manage, detect and respond to any chemical-related event that could threaten public health. Guidelines for the monitoring, assessment and management of chemical events, intoxications or poisonings should be developed and implemented after an inventory of the main sources of chemical risks has been carried out, and priorities should be established for developing emergency plans.

Gaps will be transformed into priority actions in a costed National Action Plan for Health Security (NAPHS) and addressed through domestic resources in collaboration with donors, partners and multilateral agencies. An annual review will be done to follow up the implementation of priority actions.

\section{LIMITATION}

The JEE tool used during the assessment was not pretested, this could lead to misinterpretation of some questions.

\section{Declaration}

\section{ETHICS APPROVAL AND CONSENT TO PARTICIPATE}

Not applicable

\section{CONSENT FOR PUBLICATION}


Not applicable

\section{AVAILABILITY OF DATA AND MATERIAL}

The JEE tool is accessible online at:

http://apps.who.int/iris/bitstream/handle/10665/204368/9789241510172_eng.pdf?sequence=1

\section{COMPETING INTERESTS}

The authors report no conflicts of interest. This research received no specific grant from any funding agency in the public, commercial or not-for-profit sectors.

\section{FUNDING}

Workshops organized during the process was supported by the world Health Organization, CDC country office through METABIOTA and USAID country office through the One Health Central and East Africa (OHCEA).

\section{AUTHOR'S CONTRIBUTIONS}

FNV and GO and WR conducted the JEE, MMMM, TC, SSA and AN participated in the JEE. FNV, MMMM and TC designed the study and statistical analysis as well as contributed to the drafting of the manuscript. FNV wrote the first draft and led the overall analysis. All authors contributed to the manuscript, read and approved the final manuscript.

\section{ACKNOWLEDGEMENTS}

The authors would like to appreciate the active contribution of national experts from technical departments of ministries of public health, livestock, environment, agriculture, communication, higher education, research, and defense, country partners such as the world Health Organization (WHO) country office, CDC country office through METABIOTA and USAID country office through the One Health Central and East Africa (OHCEA), the members of the international team of experts and the invaluable collaboration with governments including the governments of Mauritania, Cote d'Ivoire, Senegal, Benin, England and France, with other intergovernmental organizations, particularly experts from the WHO, the World Organization for Animal Health (OIE), the United Nations Food and Agriculture Organization (FAO), a public health institutions such as the US Centers for Disease Control (CDC) in Atlanta, private entities such as Pasteur Institute of Dakar, an independent translator/editor WHO consultant and Chesco Nogareda. The authors would also like to acknowledge the continuing support and commitment of all these actors in strengthening the implementation and values of the International Health Regulations (2005).

\section{AUTHORS' INFORMATION}


1 National Public Health Observatory Yaounde, Cameroon

2 MOSAIC, Yaounde, Cameroon

3 Ministry of health, Directorate for disease control, epidemics and pandemics, Yaounde, Cameroon

4 Virology service, Centre Pasteur of Cameroon, Yaounde, Cameroon

5 World Health Organization AFRO, WHE Programme, Dakar Hub, Senegal

6 Higher Institute of Health, Department of veterinary medicine, Université des Montagnes, Bagante, Cameroon

\section{References}

1. WHO. International Health Regulations (2005). $3^{\text {rd }}$ Edition. 2016; 1-84, http://apps.who.int/iris/bitstream/handle/10665/246107/9789241580496-eng.pdf?sequence=1 (assessed Mar 2019)

2. WHO. Implementation of the International Health Regulations (2005): Report of the Review Committee on Second Extensions for Establishing National Public Health Capacities and on IHR Implementation. 2015; 1-26.

http://apps.who.int/iris/bitstream/handle/10665/251717/B136_22Add1-en.pdf?sequence=1 (assessed Mar 2019)

3. WHO. International Health regulations (2005) Monitoring and Evaluation framwork: Joint External evaluation tool. 2016; 31-101.

http://apps.who.int/iris/bitstream/handle/10665/204368/9789241510172_eng.pdf?sequence=1 (assessed Mar 2019)

\section{Figures}



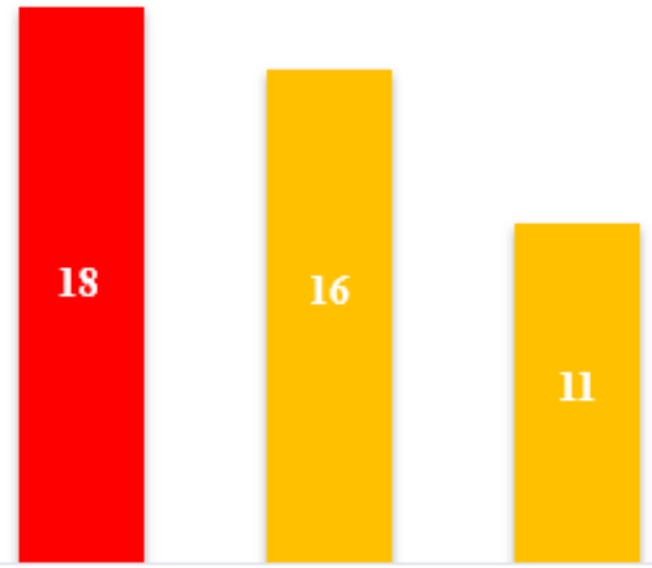

\section{3} 0

No capacity 1 Limeted Developed Demonstrated Sustainable capicity 2 capicity 3 capicity 4 capicity 5 Joint External Evaluation capacity levels

\section{Figure 1}

Number of indicators per score during the JEE $(n=48)$

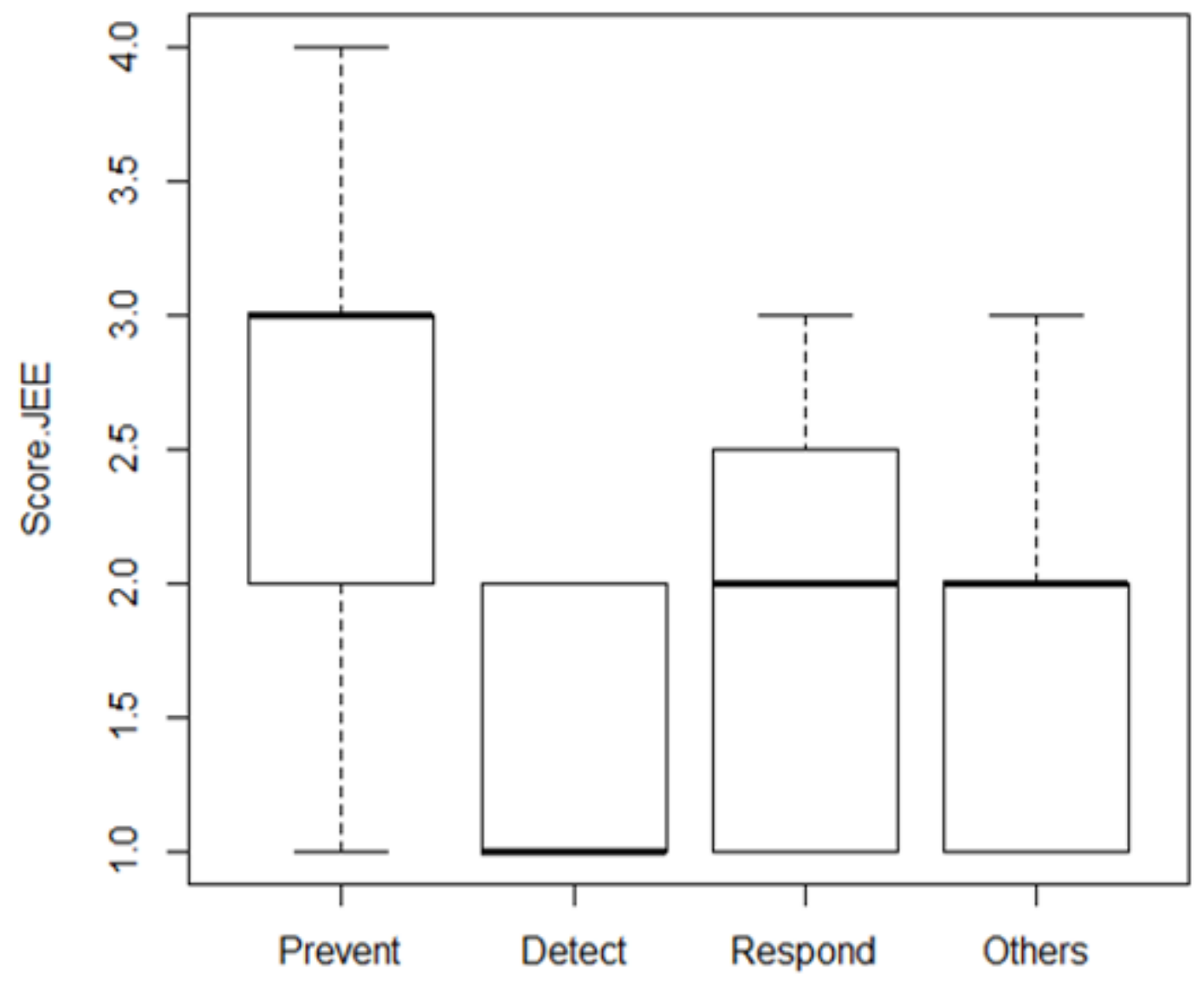

Figure 2

Dispersion of the JEE Indicators score of each domain 

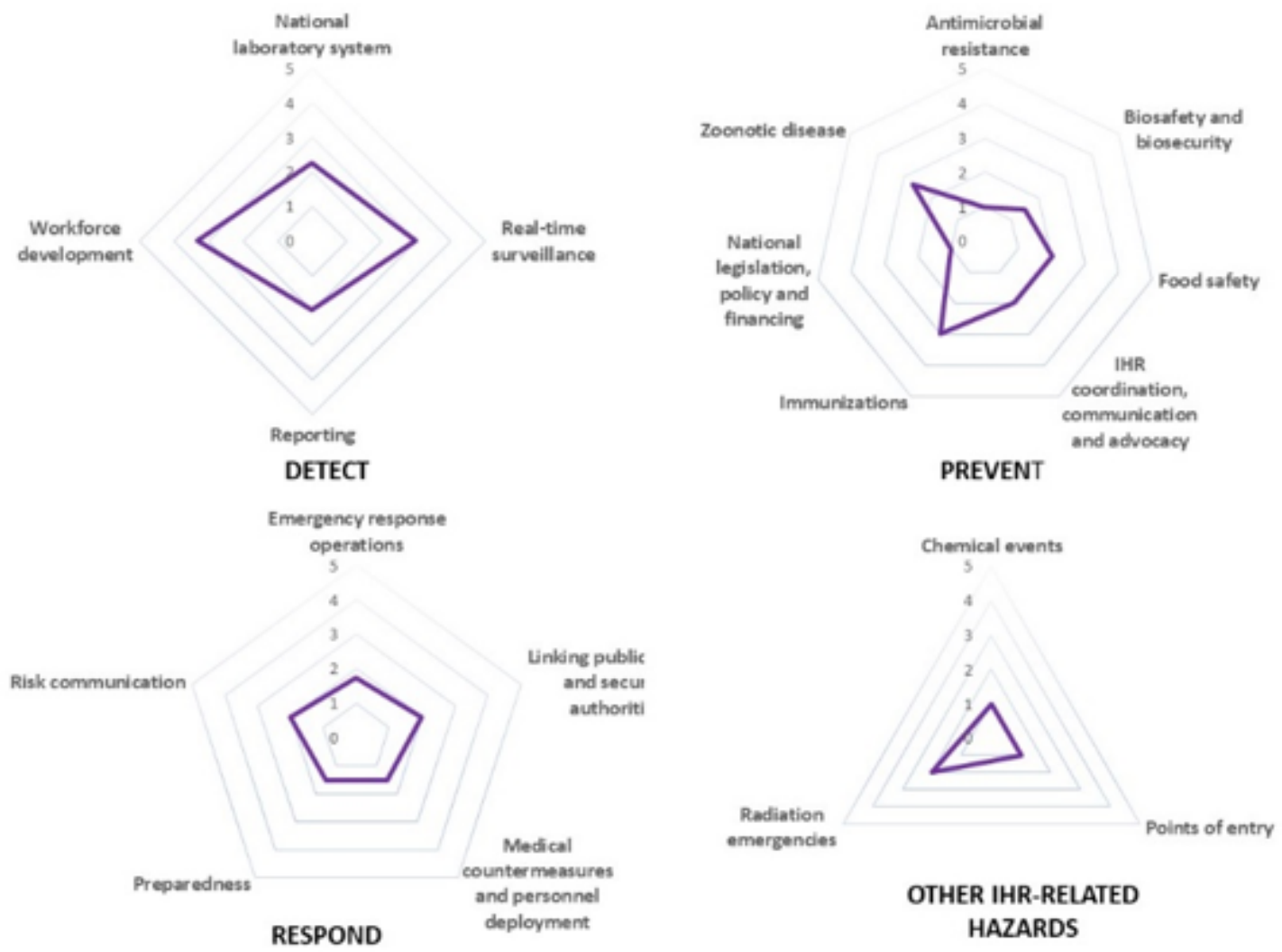

Figure 3

Summary of the JEE average score per technical areas $(n=19)$ 